\title{
Different Modeling Approaches for a Heterogeneous Liquid-Liquid Reaction Process
}

\author{
Paulo A. Quadros, Marco S. Reis, and Cristina M. S. G. Baptista* \\ GEPSI-PSE Group, Department of Chemical Engineering, University of Coimbra, \\ Pólo II, Pinhal de Marrocos, 3030-290 Coimbra, Portugal
}

\begin{abstract}
Multivariate linear regression (MLR) techniques were used to develop empirical models which are able to predict the formation of the main product and byproducts of the adiabatic benzene nitration process, as a function of the main operating conditions. Experiments carried out in a pilot plant enabled us to reproduce the operating conditions of the industrial process, providing experimental data in the intermediate and fast reaction regimes. The nitrobenzene (MNB) formation was modeled according to the film and Danckwerts mechanistic models, and the results were compared with a MLR model, showing that both approaches are suitable for describing this reaction. Nevertheless, the results stress an improved performance of the MLR model when compared to the mechanistic models, despite its structural simplicity. The statistical models developed for the nitrophenols (NPs), namely for the dinitrophenol and trinitrophenol (DNP and TNP, respectively), describe accurately the formation of these byproducts, overcoming the lack of data on kinetic and physical-chemical properties required by the mechanistic approach. The MLR models can be used for process optimization regarding conversion, productivity, and selectivity. By making use of these models, it was possible to estimate the operating conditions (temperature, $81{ }^{\circ} \mathrm{C} ; F_{\mathrm{B}} / F_{\mathrm{N}}$ ratio, 1.5 ; residence time, $1.9 \mathrm{~min}$; nitric acid concentration, 2.6\%; sulfuric acid concentration, $64 \%$; interfacial area, $46.7 \times 10^{3} \mathrm{~m}^{2} \cdot \mathrm{m}^{-3}$ ) that enable the attainment of a $99.99 \%$ MNB yield, with a total NP concentration $<215 \mathrm{ppm}$.
\end{abstract}

\section{Introduction}

The modeling of heterogeneous liquid-liquid reactions, where both mass-transfer and chemical-reaction phenomena are present, is usually a complex task, especially when it involves the development of mechanistic mathematical models. Under the mechanistic framework, two different kinds of models are usually applied: the film model, which assumes the mass transfer to be a stationary process, and the penetration models, i.e., the Higbie and the Danckwerts models, where the mass transfer is assumed to be a nonstationary phenomenon. ${ }^{1}$ The use of these models requires the estimation of several physical-chemical properties of both compounds and mixtures, as well as a number of transport and kinetic parameters, which are not always available.

Alternative empirical modeling approaches, like artificial neural networks (ANN), are used today in different fields, such as medicine ${ }^{2}$ and catalysis in chemical reaction engineering, ${ }^{3}$ among others ${ }^{4-6}$ Heterogeneous liquid-liquid reactions can also be modeled using these techniques, often requiring much less effort and time when compared to the mechanistic modeling approaches. ${ }^{7}$ Another empirical modeling technique usually used is the multivariate linear regression (MLR), which consists of establishing functional relationships among variables using a model structure that is linear in the parameters.

The mononitrobenzene, also known as nitrobenzene or $\mathrm{MNB}$, is an important starting material to produce aniline and MDI (diphenylmethane diisocyanate) polyurethanes, ${ }^{8}$ presenting an annual worldwide production

* Corresponding author. Tel.: +351-239-798738. Fax: +351239-789703. E-mail: cristina@eq.uc.pt . of near 4 million tonnes. ${ }^{9} \mathrm{~A}$ continuous adiabatic nitration process can be used to produce nitrobenzene. In this highly exothermic and heterogeneous liquid-liquid catalyzed reaction, the mass transfer assumes an important role. The reaction yield is reduced by the formation of nitrophenols (NPs), namely di- and trinitrophenol (DNP and TNP); TNP is also known as picric acid. These byproducts are harmful compounds to the environment and cannot be disposed of without a specific treatment. ${ }^{10-12}$

The search for new reactors that maximize the conversion and selectivity of this reaction process has been the main goal of recent innovations. ${ }^{13,14}$ On the other hand, the study of optimal operating conditions is also relevant and may assume an important contribution to the competitiveness of the conventional reaction processes. ${ }^{15}$ Nevertheless, for optimization to be carried out, the availability of mathematical models is fundamental. The scope of the work presented here is to build mathematical models that can be used in the identification of those sets of operating conditions that allow for the maximization of productivity, yield, and selectivity in the conventional adiabatic benzene nitration process.

\section{Multivariate Linear Regression (MLR) Modeling}

Under the heading of multivariate linear regression falls a rich variety of analysis tools, whose scope is well beyond the strict estimation of a model that is linear in its parameters. In fact, the well-established underlying theoretical background available in this field provides support and guidance through all steps of model development, including the very definition of its structure, its validation, and the detection of influential observa- 
tions, outliers, and potentially problematic data structures, namely, those related to the presence of colinearity among the regressors. Furthermore, nonlinear relationships can also be handled by this approach, as well as by dynamic models. In the multivariate linear regression models, the regression equation that expresses the relationship between the response or dependent variable, $y(i)$, and the explanatory variables $x_{j}(i)$ is given by

$$
y(i)=\beta_{0}+\beta_{1} x_{1}(i)+\beta_{2} x_{2}(i)+\ldots+\beta_{p} x_{p}(i)+u(i)
$$

where $\beta_{j}$ are the model regression coefficients for variable $x_{j}(i)$ and $u(i)$ is the random disturbance with zero mean and constant variance $\sigma^{2} .{ }^{16}$ The estimation of the model parameters is accomplished by minimizing the sum of squared residuals, i.e., using the method of least squares, leading to the estimated coefficients $b_{j}{ }^{17}$ Once $b_{j}$ has been calculated, it is possible to obtain an estimation of the observed value, $\hat{y}(i)$, using eq 2

$$
\hat{y}(i)=b_{0}+b_{1} x_{1}(i)+b_{2} x_{2}(i)+\ldots+b_{p} x_{p}(i)
$$

where the observed residual for each observation, $e(i)$, is given by

$$
\begin{aligned}
& e(i)=y(i)-\hat{\mathrm{y}}(i)= \\
& \quad y(i)-\left(b_{0}+b_{1} x_{1}(i)+b_{2} x_{2}(i)+\ldots+b_{p} x_{p}(i)\right)
\end{aligned}
$$

After estimating the parameters of the MLR model, one should proceed to its validation, according to Figure 1, where a schematic representation of the main steps involved in the development of an MLR model is presented. At this stage, the residual analysis plays an important role in the validation of the model. ${ }^{16,18}$ According to Draper and Smith, ${ }^{18}$ a model can be considered correct and precise, at a $95 \%$ confidence level, if the standardized residuals are randomly distributed about zero and confined approximately between \pm 2 . The $i$ th standardized residual, $e_{\mathrm{s}}(i)$, is obtained by dividing the residual, $e(i)$, by the estimate of the standard deviation of $u(i), s$ :

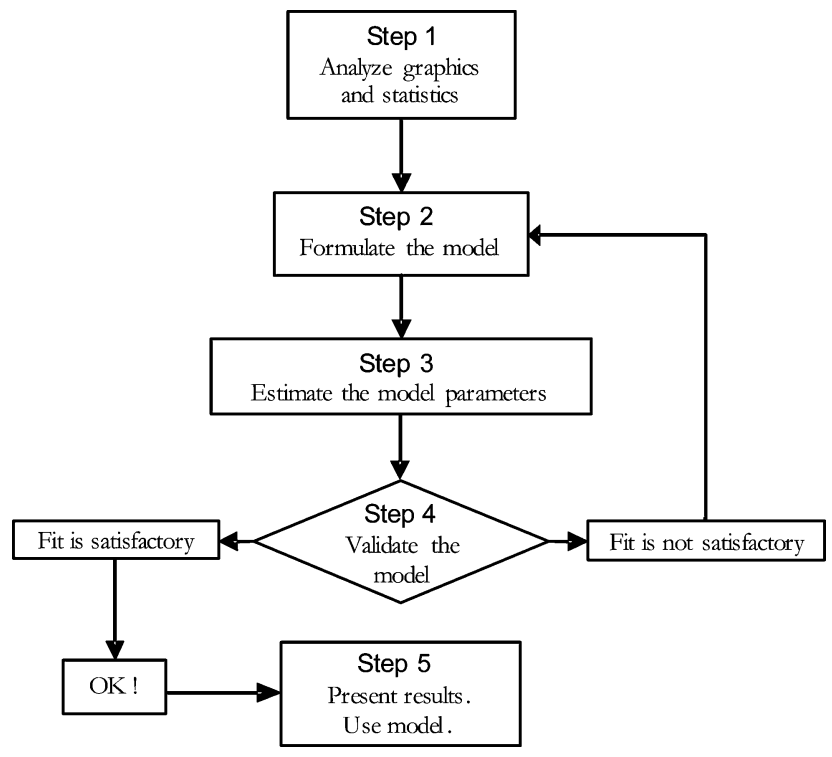

Figure 1. Multiple linear regression: Methodology.

$$
e_{\mathrm{s}}(i)=\frac{e(i)}{\left[\sum_{i=1}^{n} e^{2}(i) /(n-p-1)\right]}=\frac{e(i)}{s}
$$

The performance of the MLR model can be characterized using several criteria, like the multiple correlation coefficient, $R^{2}$, the adjusted $R$-squared, $R_{\mathrm{a}}{ }^{2}$, or the Mallows' $C_{p} \cdot{ }^{16,19,20}$ The $R^{2}$ coefficient should be as high as possible (i.e., close to unity), meaning that the model fits the data well, which essentially means that the predicted and observed values will be close to each other. However, adding variables to a model will always increase its $R^{2}$ coefficient, even when there is no relevance for predicting the variability of the response. In a limiting case, an $n$-variable model to be fitted using a set of $n$ experimental data will result in a unitary $R^{2}$ coefficient. On the other hand, the adjusted $R$-squared penalizes the $R^{2}$ metric for each new variable added, which enables one to compare the goodness of the fit when models with a different number of explanatory variables are used. The Mallows' $C_{p}$ is a measure of the total squared error that also penalizes the use of many variables and that, when a model is adequate, should have a value close to the number of predictors used.

The adequacy of a model, as well as its ability to predict unknown $y$-values, can also be quantified by calculating other measures. The root-mean-squared error (RMSE), eq 5, and the mean relative error (MRE), eq 6 , can also be used. ${ }^{21}$

$$
\begin{aligned}
\operatorname{RMSE} & =\sqrt{\sum_{i}^{n}(\hat{y}(i)-y(i))^{2} / n} \\
\mathrm{MRE} & =\frac{\sum_{i}^{n}|\hat{y}(i)-y(i)| / y(i)}{n} \times 100
\end{aligned}
$$

The RMSE underestimates the variance associated with the prediction error, since it is entirely based upon the calibration data set and does not account for the degrees of freedom used in the estimation of the model parameters. Therefore, the root-mean-squared error of calibration (RMSEC) should be used instead, as it penalizes the RMSE with the number of estimated parameters. ${ }^{21}$

$$
\operatorname{RMSEC}=\sqrt{\sum_{i}^{n}(\hat{y}(i)-y(i))^{2} /(n-p-1)}
$$

One of the main problems when developing MLR models is the multicolinearity, also known only as colinearity. This problem can be described as a correlation between two or more explanatory variables included in the model, which inflates the variance associated with the estimation of the model parameters. Colinearity cannot be seen as a modeling error in itself but rather as a condition regarding the collected data. ${ }^{16}$ This problem has natural implications in forecasting and inferences drawn from the model. A parameter that enables the quantification of colinearity is the variance inflation factor, VIF, defined by 


$$
\operatorname{VIF}\left(x_{i}\right)=\frac{1}{1-R_{i}^{2}}
$$

where $R_{i}{ }^{2}$ represents the multiple correlation coefficient of the independent variable $x_{i}$ regressed against all the remaining explanatory variables. According to Chatterjee and Price, ${ }^{16}$ if VIF assumes values $>10$, it means that colinearity is a problem. For VIF $<5$, no significant colinearity is present, and for intermediate values, some precautions should be exercised. In this range, the colinearity problems can be neglected or not, according to the model purposes. One common procedure to overcome colinearity is to not consider the potential problematic regressors by adopting a variable selection process.

Variable selection approaches evaluate which variables should be included in the model, as there are usually a lot of possible subsets of variables that can be used. According to the model purpose, e.g., control, exploratory data analysis, or forecasting, different subsets of explanatory variables can be chosen, relying on different quality criteria. Even if there are some independent variables that, because of theoretical fundamentals, should be included in the model, it is not usually possible to rely on this approach to select the most adequate subset. Another decision regards how a variable should be addressed in a model, i.e., whether it should be included in its original form or after a transformation (e.g., $x^{2}, \ln x$, or $\sqrt{x}$ ), or even as a combination of both. ${ }^{16}$ Different variable selection approaches have been proposed for building linear models; however, in this work, the forward stepwise and the best subsets regression will be used, as they present some advantages over their counterparts. ${ }^{16,18,19,22}$

\section{Continuous Adiabatic Benzene Nitration Process}

In the industry, the nitration of benzene to produce MNB is carried out either in isothermal or adiabatic processes, with the latter more commonly in use and having the advantage of utilizing the heat of this highly exothermic reaction to concentrate the sulfuric acid that is reused. The adiabatic industrial process is carried out at temperatures in the range from 100 to $140{ }^{\circ} \mathrm{C}$ with a residence time that ranges from several seconds to a few minutes..$^{13,23}$ Due to the very low solubility of benzene in the nitric and sulfuric acids, this is a heterogeneous reaction. The benzene is transferred from the organic to the aqueous phase, where it reacts with the nitronium ion, produced by the dissociation of nitric acid in the presence of sulfuric acid, which acts as a catalyst. The composition of the mixed acid, sulfuric and nitric, can be decisive to the nitric acid dissociation into nitronium ion, the real nitrating agent, ${ }^{13}$ and influences the reaction conversion and selectivity. The MNB produced is transferred to the organic phase immediately after reaction. Byproducts are formed mainly due to the limitations on the mass transfer of benzene to the aqueous phase and to the simultaneous transport of part of the undissociated nitric acid to the organic phase, where it oxidizes the benzene to form phenol. ${ }^{24}$ Once phenol is formed, it is not possible to stop its nitration. ${ }^{15}$ The concentration of sulfuric acid determines the reaction regime, which can range from a slow to a fast reaction ${ }^{25}$ and is quantified by the Hatta number, $H a{ }^{1}$
Table 1. Operating Conditions Used in the Benzene Nitration Experiments Conducted in the Pilot Plant

\begin{tabular}{ll}
\hline \multicolumn{1}{c}{ operating conditions } & \multicolumn{1}{c}{ range } \\
\hline nitration temperature, $T\left({ }^{\circ} \mathrm{C}\right)$ & $81.1-135.3$ \\
molar benzene/nitric acid feed ratio, & $0.93-1.46$ \\
$F_{\mathrm{B}} / F_{\mathrm{N}}$ & \\
stirring speed, $n(\mathrm{rpm})$ & $394-1700$ \\
interfacial area, $a\left(\mathrm{~m}^{2} \mathrm{~m}^{-3}\right)$ & $580.4-58044.5$ \\
residence time, $\tau\left(\mathrm{min}^{-}\right)$ & $1.93-6.06$ \\
volumetric phase ratio, $Q_{\mathrm{aq}} / Q_{\mathrm{org}}$ & $5.37-15.79$ \\
holdup fraction, $\epsilon$ & $0.060-0.157$ \\
nitric acid content in the mixed acid, & $2.61-6.39$ \\
$\quad N($ wt $\%)$ & \\
sulfuric acid content in the mixed acid, & $57.41-68.90$
\end{tabular}
$S($ wt $\%)$

\section{Experimental Section}

The pilot plant and the operating procedure described in the literature ${ }^{15}$ were used to perform a series of benzene nitration experiments, in a broad range of operating conditions, close to the ones used in the benzene adiabatic industrial nitration process, as shown in Table 1. Previous experiments performed in this pilot plant were carried out in the intermediate reaction regime, ${ }^{26}$ with $0.3<H a<2$. To gather more information on this process and, in particular, on the relevance of some operating conditions, new experiments were performed. Wider ranges of residence times and molar feed ratios between benzene and nitric acid $\left(F_{\mathrm{B}} / F_{\mathrm{N}}\right)$ as well as different mixed acid compositions were used. These new experiments were carried out in both the intermediate and fast reaction regimes, embracing Hatta numbers in the range $0.3<H a<3.5$. Thus, it was possible to obtain a large number of experimental data on the formation of MNB and NPs, with a total of $\sim 150$ experimental data points, in the intermediate and the fast reaction regimes, as illustrated in Figure 2. It is

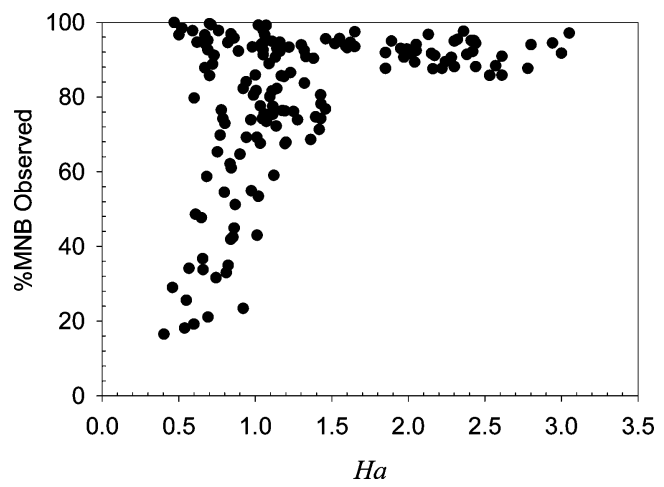

Figure 2. Hatta number versus observed MNB production.

noticeable that the same MNB production can be obtained for a wide range of Hatta numbers. This shows that the Hatta number alone cannot explain the production of MNB. Moreover, different operating conditions may lead to different Hatta numbers but to the same MNB production. This result was already addressed and enlightened in previous work. ${ }^{26}$ Thus, a complete analysis of all the influential operating conditions should be carried out in order to get more insight into this heterogeneous chemical process.

\section{Nitrobenzene Formation Modeling: Mechanistic Versus MLR Models}

5.1. Mechanistic Approach: Film and Danckwerts Models. The film and the penetration Danck- 
werts models are usually used to mechanistically describe the heterogeneous fluid-fluid reactions. ${ }^{1}$ To get more information on what influences, in particular, the benzene nitration under industrially relevant operating conditions, these models have already been applied to the intermediate reaction regime with success. ${ }^{26,27} \mathrm{In}$ this regime, no significant differences have been detected between the full solution of the film model and the Danckwerts solution. ${ }^{27}$ However, the fast reaction regime under these operating conditions was not previously tested. The models developed in Quadros et al. ${ }^{27}$ were applied to these new sets of experimental data, and the results of their predictions are presented in Figure 3 . The adequacy of both models to describe this heterogeneous liquid-liquid reaction, in both the intermediate and fast reaction regimes, is clearly shown in this figure. No significant differences are detected between the two models. All the prediction results are comprised within a $\pm 15 \%$ range for both models and reaction regimes.

5.2. Empirical Statistical Approach: Multivariate Linear Regression Model. As expressed previously, to avoid problems such as multicolinearity, one should decide which set of explanatory variables to use for building an MLR model. Table 2 shows a summary of the colinearity analysis performed on the full set of explanatory variables to be included in the models. The analysis is based on the variation inflation factor VIF, eq 8 , and the procedure consists of eliminating, at each step, one of the variables that presents a higher VIF or a known relationship with other variables. In this process, the stirring speed and the holdup fraction have a major influence on the effective interfacial area; ${ }^{28}$ thus, only the effective interfacial area was selected to enter the model, to keep this modeling approach comparable with the mechanistic ones, which also assume the explicit knowledge of this parameter. If this model, based on the effective interfacial area, presents better predictive performance versus the mechanistic alterna-

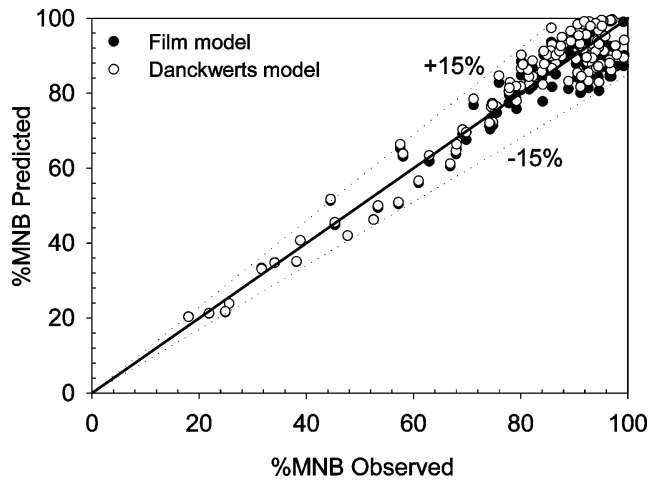

Figure 3. Film and Danckwerts models for the MNB production versus the MNB observed production.

Table 2. Selection of the Explanatory Variables To Be Included in the Modeling Process

\begin{tabular}{|c|c|c|c|c|c|c|}
\hline \multirow[b]{2}{*}{ variable } & \multicolumn{2}{|c|}{ 1st step } & \multicolumn{2}{|c|}{ 2nd step } & \multicolumn{2}{|c|}{ 3rd step } \\
\hline & $R^{2}$ & VIF & $R^{2}$ & VIF & $R^{2}$ & VIF \\
\hline$T$ & 0.7517 & 4.03 & 0.5640 & 2.29 & 0.5609 & 2.28 \\
\hline$F_{\mathrm{B}} / F_{\mathrm{N}}$ & 0.8831 & 8.55 & 0.8781 & 8.20 & 0.3329 & 1.50 \\
\hline$n$ & 0.8930 & 9.35 & & & & \\
\hline 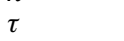 & 0.3088 & 1.45 & 0.2882 & 1.40 & 0.2785 & 1.39 \\
\hline$N$ & 0.9787 & 46.98 & 0.9753 & 40.43 & 0.7348 & 3.77 \\
\hline$S$ & 0.8358 & 6.09 & 0.8205 & 5.57 & 0.7486 & 3.98 \\
\hline$Q_{\mathrm{aq}} / Q_{\mathrm{org}}$ & 0.9618 & 26.20 & & & & \\
\hline $8 x_{1}$ & 0.9792 & 48.03 & 0.5 & 34.1 & & \\
\hline$a$ & 0.9032 & 10.33 & 0.6990 & 3.32 & 0.6595 & 2.94 \\
\hline
\end{tabular}

tives, then one also has justified the expectations that a model involving the manipulated variables (stirring speed and the holdup fraction) would also perform better. This was verified based on results that relate the interfacial area to the stirring speed and the holdup fraction, ${ }^{28}$ although those results are not presented in this paper. Despite the higher VIF obtained for the nitric acid concentration in the mixed acid, in the first and second selection steps, this variable was kept in the model building process, because of its acknowledged contribution to the reaction conversion and selectivity. ${ }^{13}$ To eliminate the colinearity problems that arise from considering the nitric acid concentration, the volumetric ratio between the phases was withdrawn, as this effect is somehow taken into account by the effective interfacial area. Table 2 shows that the final subset of regressors does not present colinearity problems, since all the VIF values are $<5$.

The software package Statistica $6^{19}$ was used to implement the forward stepwise and the best subsets regression methods in the development of the MLR models. Variables transformations were considered as indicated in the Introduction section. An iterative procedure was used to build up each model, considering only the more significant explanatory variables. At each step of model buildup, the less significant variable was deleted. Criteria like the student $t$-test and the $p$-level were used to evaluate the variables' significance. ${ }^{16}$ The logarithmic transformation of the dependent variable was also considered. Outliers and residual analysis were performed at each step. More details regarding the adopted MLR model development process can be found elsewhere. ${ }^{29}$ In Figure 4 the nitrobenzene production predicted by the MLR model is represented versus the observed value. A good agreement is achieved between the observed and the predicted values. Similar to the mechanistic models, all predictions are comprised within a $\pm 15 \%$ error range. For the nitrobenzene production, the best equation found uses four explanatory variables, as indicated in eq 9, which were selected according to their explanation ability. It is possible to observe that the influential variables in the MNB production are the interfacial area, the sulfuric and nitric acid concentrations, and the temperature. The increase in the nitric acid concentration leads to a decrease in the MNB formation, while an increase in the other parameters augments the MNB production.

$$
\begin{aligned}
& \mathrm{MNB}[\%]=14.2450 \ln (a)+85.8979 \ln (S)+ \\
& 28.6730 \ln (T)-12.5394 \ln (N)-526.65
\end{aligned}
$$

A total of 144 experimental data points were used to obtain the MNB model, leading to an adjusted correla-

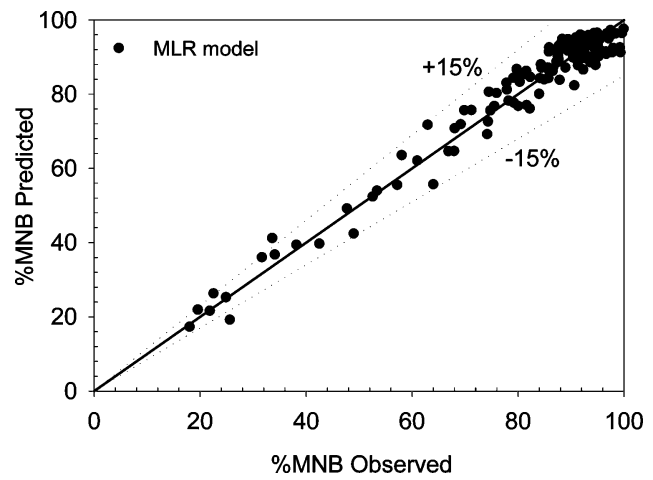

Figure 4. MNB production by the MLR model versus the observed MNB production. 
Table 3. MNB Models' Results

\begin{tabular}{|c|c|c|c|c|c|}
\hline & \multicolumn{2}{|c|}{$\begin{array}{l}\text { mechanistic } \\
\text { approach }\end{array}$} & \multicolumn{3}{|c|}{$\begin{array}{c}\text { empirical } \\
\text { statistical approach }\end{array}$} \\
\hline & $\begin{array}{l}\text { film } \\
\text { model }\end{array}$ & $\begin{array}{c}\text { Danckwerts } \\
\text { model }\end{array}$ & $\begin{array}{l}\text { full sample } \\
\text { model }\end{array}$ & $\begin{array}{c}\text { training } \\
\text { sample model }\end{array}$ & $\begin{array}{c}\text { test } \\
\text { sample }\end{array}$ \\
\hline & $138^{a}$ & 13 & $144^{b}$ & $115^{b}$ & $29^{c}$ \\
\hline RMS & $5.41^{c}$ & $5.17^{c}$ & $3.90^{d}$ & $3.86^{d}$ & $4.19^{c}$ \\
\hline MRE (\%) & 5.49 & 5.57 & 4.32 & 4.25 & 4.96 \\
\hline
\end{tabular}

${ }^{a}$ Number of data points used to test the model. ${ }^{b}$ Number of data points used to estimate the model. ${ }^{c}$ RMSE. ${ }^{s}$ RMSEC.

tion coefficient $\left(R_{\mathrm{a}}{ }^{2}\right)$ of 0.9578 , a root-mean-squared error calibration (RMSEC) of 3.90, and mean relative error (MRE) of $4.32 \%$, as given in Table 3 .

An additional validation step for the nitrobenzene MLR model was performed by a crosscheck validation analysis. The experimental data were divided into two different sets: a training and a test sample. The training sample was used to obtain the model in eq 10 , while the test sample was used to validate its performance. The training and test samples show performance measures identical to the model developed using all samples, indicating its validity as discussed in the next section. In future calculations, eq 10 will be used to estimate the MNB concentration.

$$
\begin{aligned}
& \mathrm{MNB}[\%]=14.7933 \ln (a)+79.4166 \ln (S)- \\
& 12.7082 \ln (N)+22.9980 \ln (T)-478.24
\end{aligned}
$$

5.3. Comparison between the Mechanistic and the MLR Models' Results. After presenting both modeling approaches for the MNB production, as well as their results, we will now compare their performance. Even though it has been shown in Figures 3 and 4 that both modeling approaches are suitable for modeling this heterogeneous liquid-liquid reaction, we would like to point out that the results do show some differences. Table 3 presents the results for the methodologies used, namely the RMSE and the MRE, and the number of data used to build (full sample and training sample models) or test the model (film and Danckwerts models and test sample). For the statistical models, the rootmean-squared error calculated was the RMSEC, which accounts for the number of model parameters.

In Table 3 , it is clear that the MLR models present lower error measures than the mechanistic film and Danckwerts models. Results also show that the training set led to a precise MLR model, with good prediction accuracy confirmed by the test sample errors results, which are lower than the errors obtained for the mechanistic models. The use of an MLR model enables one to observe the direct influence of a given explanatory variable on the dependent one, whereas for the mechanistic models, this kind of sensitive analysis can be rather involved. Thus, in this case study, the MLR techniques proved to be much less time consuming and more accurate.

\section{Nitrophenols' Formation Modeling}

The present lack of knowledge on both mass transfer and kinetic parameters for the nitrophenols' formation, as side reactions of the benzene nitration, prohibits the use of the mechanistic modeling approaches. Once again, the MLR methodology is an alternative for describing these side reactions. This section presents the results for the MLR models developed and validated regarding the DNP and TNP formation.
6.1. Multivariate Linear Regression Model for Dinitrophenol (DNP). Identical procedures to those described in the previous section were used to estimate an MLR model for the DNP formation as a function of the main process operating conditions. Thus, after obtaining a first model, the experimental data sample was divided into a training and a test sample. ${ }^{29}$ The model obtained with the training sample is given by eq 11. The influential variables in the DNP formation are the effective interfacial area, the temperature, and the molar feed ratio between the benzene and nitric acid.

$$
\begin{aligned}
& \ln (\mathrm{DNP}[\mathrm{ppm}])=0.1937 \ln (a)+6.4744 \ln (T)- \\
& \quad 0.2476\left(F_{\mathrm{B}} / F_{\mathrm{N}}\right)^{2}-\left(1.45 \times 10^{-4}\right) T^{2}-23.6576
\end{aligned}
$$

Table 4 shows the error measures associated with this model. Low mean relative errors are obtained for the DNP model, and because the errors reported for the training and for the test sample are very similar, the model adequacy is confirmed. This observation is also supported by Figure 5, where the predicted versus observed values for $\ln (\mathrm{DNP})$ are plotted for the training sample model and for the test sample.

6.2. Multivariate Linear Regression Model for Trinitrophenol (TNP). The model for the TNP obtained with the training set is given by eq 12 . Contrary to the previous models, this one uses every noncolinear explanatory variable in Table 2 . This indicates that the TNP formation depends on more operating conditions than the other reaction products, such as, for instance, the residence time, which has no contribution in either the MNB or the DNP models.

$$
\begin{array}{r}
\ln (\mathrm{TNP}[\mathrm{ppm}])=\left(4.00 \times 10^{-4}\right) T^{2}+14.5065 \ln (S)+ \\
3.2623 \ln (N)-\left(3.32 \times 10^{-5}\right) a-1.6566 \ln \left(F_{\mathrm{B}} / F_{\mathrm{N}}\right)- \\
0.0118 \tau^{2}-64.4487(12)
\end{array}
$$

\begin{tabular}{|c|c|c|c|c|}
\hline & \multicolumn{2}{|l|}{ DNP } & \multicolumn{2}{|l|}{ TNP } \\
\hline & $\begin{array}{c}\text { training } \\
\text { sample model }\end{array}$ & $\begin{array}{c}\text { test } \\
\text { sample }\end{array}$ & $\begin{array}{c}\text { training } \\
\text { sample model }\end{array}$ & $\begin{array}{c}\text { test } \\
\text { sample }\end{array}$ \\
\hline$n$ & $111^{b}$ & $28^{c}$ & $106^{b}$ & $24^{c}$ \\
\hline $\mathrm{RMSE}^{a}$ & $73.82^{d}$ & $68.19^{e}$ & $50.61^{d}$ & $49.64^{e}$ \\
\hline $\operatorname{MRE}(\%)$ & 6.76 & 5.37 & 16.53 & 13.10 \\
\hline
\end{tabular}

The $\ln (\mathrm{TNP})$ results for the training sample model and for the test sample are shown in Figure 6. The errors

Table 4. DNP and TNP Models' Results.

${ }^{a}$ These errors are reported to the DNP and TNP values obtained by the respective logarithmic models and not to the $\ln (\mathrm{DNP})$ or $\ln$ (TNP) values. ${ }^{b}$ Number of data points used to estimate the model. ${ }^{c}$ Number of data points used to test the model. ${ }^{d}$ RMSEC. e RMSE.

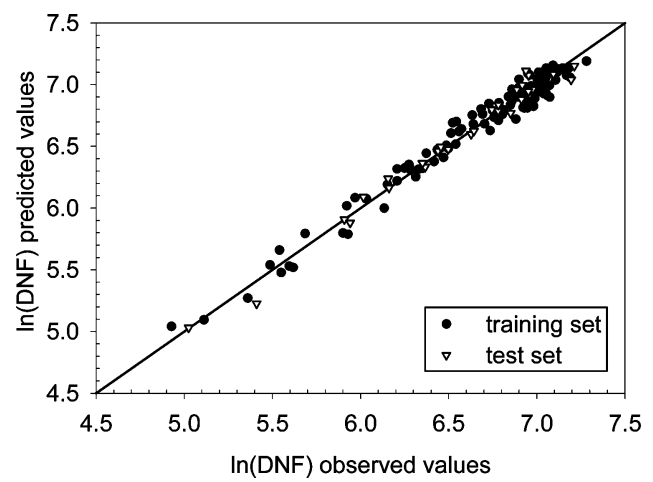

Figure 5. Predicted versus observed values for the $\ln (\mathrm{DNP})$ model: Training and test sets. 


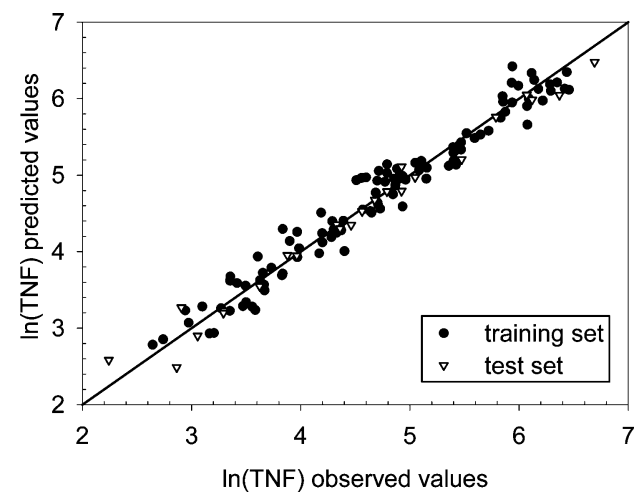

Figure 6. Predicted versus observed values for the $\ln (\mathrm{TNP})$ model: Training and test sets.

obtained for this model are given in Table 4, where it is possible to observe that the RMSE for the TNP are lower than the errors obtained for the DNP, while the opposite happens for the MRE. This can be explained by the magnitude of the concentrations of both compounds: the DNP concentration is always higher than that of the TNP, leading to a higher mean squared error due to the higher absolute values but with a lower mean relative error.

\section{Operating Conditions Process Optimization}

The MLR models obtained can now be used for a sensitive analysis concerning the influence of the process operating conditions on the production of MNB and NPs. The standard operating conditions considered are comprised in the range of the experiments performed and summarized in Table 5. To evaluate its impact on

Table 5. Standard Operating Conditions Considered in Process Optimization

$\begin{array}{llll}T W & 100.0{ }^{\circ} \mathrm{C} & N & 4.0 \% \\ F_{\mathrm{B}} / F_{\mathrm{N}} & 1.0 & S & 57.4 \% \\ \tau & 2.0 \min & a & 30000 \mathrm{~m}^{2} \cdot \mathrm{m}^{-3}\end{array}$

the concentration of the reaction products, each operating condition was increased by $20 \%$ versus its standard value. The MLR models developed were used to calculate the relative variance on each reaction product concentration, relative to the $20 \%$ increase in each operating condition. The results are given in Figure 7, and they show that the most influential operating conditions in this process are the reaction temperature and the sulfuric and nitric acid concentrations. The TNP concentration is particularly sensitive to a $20 \%$ increase in temperature and sulfuric acid concentration, since its concentration is augmented by about 500 and $1300 \%$,

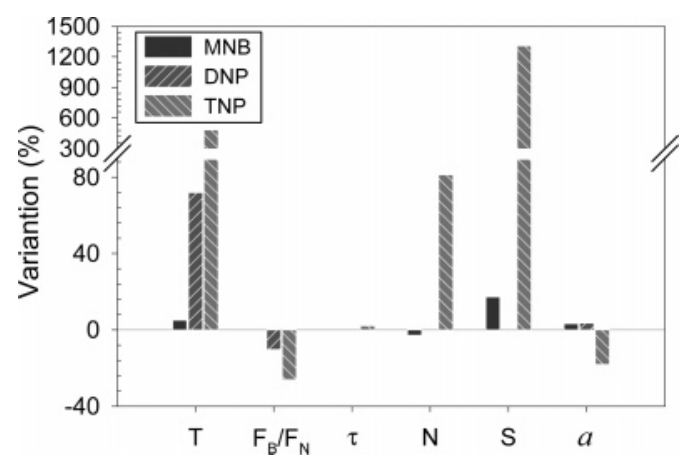

Figure 7. MNB, DNP, and TNP variation observed (\%) for a $20 \%$ increase in each explanatory variable. respectively. Figure 7 also shows that an increase in the $F_{\mathrm{B}} / F_{\mathrm{N}}$ ratio produces a decrease in the NPs concentration and does not influence the final MNB concentration. The effective interfacial area increase leads to a decrease in TNP concentration and to a slight increase in the MNB and DNP concentrations. These conclusions confirm those in the literature ${ }^{15}$ however, here a quantitative assessment is done. The residence time is not a very influential variable in the process, increasing the TNP concentration only slightly.

In the next sections, the MLR models developed are used to optimize the operating conditions in the benzene nitration process, regarding the maximization of the conversion and selectivity. Two scenarios are considered, Case Study 1 and Case Study 2, with the second being less restrictive in what concerns the operating ranges. This leads to the enunciation of a linear problem, solved by the Solver tool provided by the Microsoft Excel program. The objective function was the minimization of the total concentration of NPs, under the constraints presented in each case.

7.1. Case Study 1. The ranges of the operating conditions were set according to the practices of the industrial adiabatic benzene nitration process. Table 6 shows the boundaries imposed on each operating variable, as well as on the reaction products, used in this optimization case. A high conversion of MNB was set as priority (99.99\%), while the highest total NPs concentration allowed was fixed at $2500 \mathrm{ppm}$. The solution of this optimization problem, in Table 7 , revealed that it was not possible to verify all the constraints simultaneously, namely the $99.99 \%$ MNB. The highest MNB conversion attained was $98.67 \%$, with a concentration of NPs close to $1550 \mathrm{ppm}$. It is worth noticing that the optimal solution forces all the variables to reach its boundaries, which prevents the attainment of the product concentrations established in Table 6, in particular, the targeted MNB concentration. A highconcentration of NPs was obtained, which may be due to the high temperature $\left(135^{\circ} \mathrm{C}\right)$ in the reactor. It is known that an increase in temperature causes a considerable rise in the NP concentration, ${ }^{15}$ because the activation energies for the NP reactions are high when compared to that for the MNB formation reaction. ${ }^{30}$ Therefore, broader ranges of operating conditions should be used to meet all the restrictions imposed.

7.2. Case Study 2. On the basis of the information collected in case study 1 , broader ranges of operating

Table 6. Boundaries Imposed on the Operating Conditions and Reaction Products Concentration-Case Study 1

$\begin{array}{lll}130 & <T< & 135 \\ 1.06 & <F_{\mathrm{B}} / F_{\mathrm{N}}< & 1.1 \\ 1.9 & <\tau<< & 2.1 \\ 4.5 & <N< & 5.5 \\ 57.5 & <S< & 58.5 \\ 10000 & <a< & 50000 \\ 99.99 & <\mathrm{MNB}< & 100 \\ 0 & <\mathrm{DNP}< & 2000 \\ 0 & <\mathrm{TNP}< & 500 \\ 0 & <\mathrm{NPS}< & 2500\end{array}$

Table 7. Optimization Results-Case Study 1

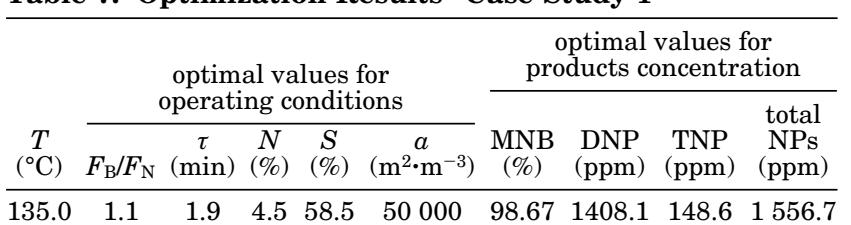


Table 8. Boundaries Imposed on the Operating Conditions and Reaction Products Concentration-Case Study 2.

$\begin{array}{lll}80 & <T< & 135 \\ 0.93 & <F_{\mathrm{B}} / F_{\mathrm{N}}< & 1.5 \\ 1.9 & <\tau<< & 6 \\ 2.6 & <N< & 6.4 \\ 57.4 & <S< & 64 \\ 10000 & <a< & 50000 \\ 99.99 & <\mathrm{MNB}< & 100 \\ 0 & <\mathrm{DNP}< & 250 \\ 0 & <\mathrm{TNP}< & 5 \\ 0 & <\mathrm{NPS}< & 250\end{array}$

conditions were used in this second case study, and these are summarized in Table 8. Lower temperatures and nitric acid concentrations and higher $F_{\mathrm{B}} / F_{\mathrm{N}}$ ratios and nitric and sulfuric acid concentrations were now allowed. Table 9 shows the set of optimal operating

Table 9. Optimization Results-Case Study 2

\begin{tabular}{|c|c|c|c|c|c|c|c|c|c|}
\hline \multirow[b]{3}{*}{$\begin{array}{c}\mathrm{T} \\
\left({ }^{\circ} \mathrm{C}\right)\end{array}$} & \multirow{2}{*}{\multicolumn{5}{|c|}{$\begin{array}{l}\text { optimal values for } \\
\text { operating conditions }\end{array}$}} & \multicolumn{4}{|c|}{$\begin{array}{l}\text { optimal values for } \\
\text { products concentration }\end{array}$} \\
\hline & & & & & & \multirow[b]{2}{*}{$\underset{(\%)}{\operatorname{MNB}}$} & \multirow[b]{2}{*}{$\begin{array}{l}\text { DNP } \\
(\mathrm{ppm})\end{array}$} & \multirow[b]{2}{*}{$\begin{array}{c}\text { TNP } \\
(\mathrm{ppm})\end{array}$} & \multirow{2}{*}{$\begin{array}{l}\text { total } \\
\text { NPs } \\
(\mathrm{ppm})\end{array}$} \\
\hline & $F_{\mathrm{B}} / F_{\mathrm{N}}$ & $\underset{\tau}{\tau}(\min )$ & $\begin{array}{l}N \\
(\%)\end{array}$ & $\underset{(\%)}{S}$ & $\begin{array}{c}a \\
\left(\mathrm{~m}^{2} \mathrm{~m}^{-3}\right)\end{array}$ & & & & \\
\hline 0.9 & 1.5 & 1.9 & 2.6 & 64.0 & 46666 & 99.99 & 213.4 & 0.6 & 214.0 \\
\hline
\end{tabular}

conditions obtained, as well as the product and byproduct concentrations that resulted. These larger ranges enabled the achievement of the desired concentrations. The optimal values for the temperature, the nitric acid concentration, and the residence time are very close to their lower limit $\left(\sim 81{ }^{\circ} \mathrm{C}, 2.6 \%\right.$ and $\left.1.9 \mathrm{~min}\right)$, while the other operating parameters nearly reach the maximum values considered in Table 8 . The striking feature in the outcome of this optimization study is that the desired $99.99 \%$ MNB concentration is reached with an amazing reduction on the NP concentration which, in total, does not exceed a level of $214 \mathrm{ppm}$. It is also interesting to notice that only traces of TNP were obtained, thus confirming previous experiments where no TNP was detected at temperatures $<85{ }^{\circ} \mathrm{C} .15$

These two examples illustrate, in clear terms, how the estimated MLR model can be used in the important context of optimizing the operation conditions. However, in practice, the procedure should be formulated in terms of the manipulated variables available, after their integration in a MLR model, to facilitate the necessary adjustment actions to be operated by the process personal and to reduce the interaction among the model input variables.

To sum up, these MLR models allow one to evaluate the benzene nitration products concentration and to perform a simultaneous optimization of the process conversion and selectivity, using a large range of operating conditions. Moreover, these models' results showed that an important reduction in the byproducts formation is achievable, without putting at risk the process conversion, just by changing some of the present operating conditions to a range already tested at a pilot plant level. These changes in the operating conditions may also be performed in an industrial plant, with no additional equipment or expensive costs, provided scaleup problems do not occur. Therefore, the work presented here may contribute to an important decrease in the industrial effluent to be treated, with associated economical and environmental benefits.

\section{Conclusions}

The main goal of this work was to obtain MLR models to describe the concentration of MNB as well as that of the benzene nitration byproducts, DNP, and TNP, as a function of the main operating conditions. Experimental runs were performed in a pilot plant reactor that allows the simulation of the industrial operating conditions ranges. The results of these experiments were used to develop MLR models for each product or byproduct.

The nitrobenzene MLR model results were compared to those obtained with the mechanistic film and Danckwerts models, showing this model's adequacy and better performance. Moreover, the MLR models proved to be easier to obtain, because no kinetic or mass transfer parameters, or even physical-chemical properties, need to be estimated.

The MLR models were used in two optimization case studies in order to identify the range of operating conditions that enable one to accomplish target values for the conversion and selectivity of the process. The optimization results point out that a low temperature $\left(81^{\circ} \mathrm{C}\right)$, nitric acid concentration $(2.6 \%)$, and residence time (1.9 $\mathrm{min})$ should be used while, on the other hand, a high benzene-to-nitric-acid molar feed ratio (1.5), interfacial area $\left(46.7 \times 10^{3} \mathrm{~m}^{2} \cdot \mathrm{m}^{-3}\right)$, and sulfuric acid concentration (64\%) must be employed to perform a process optimization. These conditions are in the range of the experiments performed in the pilot plant but were not yet tested in an industrial plant. Nevertheless, it is expected that these operating condition ranges may, in the future, provide economical and environmental benefits to the adiabatic benzene nitration plants.

\section{Acknowledgment}

Financial support from Fundação para a Ciência e a Tecnologia (FCT), for the Ph.D Grant SFRH/BD/1266/ 2000, and from Quimigal S.A., Portugal, is gratefully acknowledged. We also acknowledge the assistance of Ângela Sousa and Hugo Silva in the experimental work, as well as Pedro Francisco in the MLR modeling techniques.

\section{Notation}

$a=$ effective interfacial area, $\left(\mathrm{m}^{2} \mathrm{~m}^{-3}\right)$

$b=$ estimation of the model partial regression coefficients

$e=$ observed residual for each observation

$e_{\mathrm{s}}=$ standardized residual

$F_{\mathrm{B}} / F_{\mathrm{N}}=$ molar feed ratio between benzene and nitric acid

$\mathrm{Ha}=$ Hatta number

$n=$ number of data points

$n=$ stirring speed, rpm

$N=$ nitric acid content in the mixed acid, wt \%

$p=$ number of explanatory variables included in the model

$Q_{\text {aq }} / Q_{\text {org }}=$ volumetric phase ratio

$s=$ estimate of standard deviation

$S=$ sulfuric acid content in the mixed acid, wt \%

$T=$ nitration temperature,${ }^{\circ} \mathrm{C}$

$u=$ random disturbance

$\mathrm{VIF}=$ variance inflation factor

$x=$ independent variable

$y=$ dependent variable

$\hat{y}=$ estimation of the observed value

Greek Letters

$\beta=$ standardized model partial regression coefficients

$\epsilon=$ holdup fraction 
$\sigma^{2}=$ variance

$\tau=$ residence time, $\min$

\section{Literature Cited}

(1) Westerterp, K. R.; Van Swaaij, W. P. M.; Beenackers, A. A. C. M. Chemical reactor design and operation, 2nd ed.; John Wiley \& Sons: Chichester, U.K., 1990.

(2) Kuzmanovski, I.; Ristova, M.; Soptrajanov, B.; Stefov, V.; Popovski, V. Determination of the composition of sialoliths composed of carbonate apatite and albumin using artificial neural networks. Talanta 2004, 62, 813-817.

(3) Serra, J. M.; Corma, A.; Chica, A.; Argente, E.; Botti, V. Can artificial neural networks help the experimentation in catalysis? Catal. Today 2003, 81, 393-403.

(4) Yu, D. L.; Gomm, J. B.; Williams, D. Sensor fault diagnosis in a chemical process via RBF neural networks. Control Eng. Pract. 1999, $7,49-55$.

(5) Edwards, P. J.; Murray, A. F.; Papadopoulos, G.; Wallace, A. R.; Barnard, J.; Gordon, S. Paper curl prediction and control using neural networks. Tappi J. 1999, 82 (7), 145-151.

(6) Elkamel, A.; Abdul-Wahab, S.; Bouhamra, W.; Alper, E. Measurement and prediction of ozone levels around a heavily industrialized area: a neural network approach. Adv. Environ. Res. 2001, 5, 47-59.

(7) Molga, E.; Cherbanski, R. Catalytic reaction performed in liquid-liquid system: comparison of conventional and neural networks modelling methods. Catal. Today 2003, 79-80, 241247.

(8) Eiermann, M.; Ebel, K. Nitration of aromatic hydrocarbons. U.S. Patent 6,362,381, 2002.

(9) Haas, C. Aniline the builder. Process technology: Aniline. Eur. Chem. News 2004, 1-7 (March), 16-18.

(10) Adams, E. G.; Barker, R. B. Process for extracting and disposing of nitrophenolic byproducts. U.S. Patent 4,925,565, 1990

(11) Boyd, D. A.; Gairns, S. A.; Guenkel, A. A. Integrated effluent treatment process for nitroaromatic manufacture. U.S. Patent 6,288,289, 2001.

(12) Zhang, S.; Arcangeli, J. P.; Livingston, A. G.; Boam, A. T. Process for removing and recovering of phenolic compounds from aqueous fluids. U.S. Patent 6,586,638, 2003.

(13) Guenkel, A. A.; Rae, J. M.; Hauptmann, E. G. Nitration process. U.S. Patent 5,313,009, 1994.

(14) Burns, J. R.; Ramshaw, C. A microreactor for the nitration of benzene and toluene. Chem. Eng. Commun. 2002, 189, 16111628.

(15) Quadros, P. A.; Castro, J. A. A. M.; Baptista, C. M. S. G. Nitrophenols reduction in the benzene adiabatic nitration process. Ind. Eng. Chem. Res. 2004, 43, 4438-4445.
(16) Chatterjee, S.; Price, B. Regression analysis by example, 2nd ed.; John Wiley \& Sons: New York, 1991.

(17) Davies, O. L.; Goldsmith, P. L. Statistical methods in research and production, 4th ed.; Oliver \& Boyd: Edinburgh, U.K., 1972.

(18) Draper, N. R.; Smith, H. Applied regression analysis. John Wiley \& Sons: New York, 1966.

(19) STATISTICA (data analysis software system), version 6, electronic manual; StatSoft, Inc.: Tulsa, OK, 2003; www.statsoft.com.

(20) Garson, G. D. Multiple regression. Statnotes: An online textbook; 2004, http://www2.chass.ncsu.edu/garson/regress.htm.

(21) Naes, T.; Isaksson, T.; Fearn, T.; Davies, T. A user-friendly guide to multivariate calibration and classification; NIR Publications: Chichester, U.K., 2002.

(22) Guimarães, R. C.; Cabral, J. A. S. Estatística; Ed. Revista, McGraw-Hill: Lisboa, Portugal, 1997.

(23) Alexanderson, V.; Trecek, J.; Vanderwaart, C. Continuous adiabatic process for the mononitration of benzene. U.S. Patent 4,091,042, 1978.

(24) Burns, J. R.; Ramshaw, C. Development of a microreactor for chemical production. Trans. Inst. Chem. Eng. A 1999, 77, 206211.

(25) Cox, P. R.; Strachan, A. N. Two-phase nitration of toluene, Part II. Chem. Eng. J. 1972, 4, 253-261.

(26) Quadros, P. A.; Oliveira, N. M. C.; Baptista, C. M. S. G. Continuous adiabatic industrial benzene nitration with mixed acid at a pilot plant scale. Chem. Eng. J. 2005, 108, 1-11.

(27) Quadros, P. A.; Oliveira, N. M. C.; Baptista, C. M. S. G. Benzene nitration: validation of heterogeneous reaction models. Chem. Eng. Sci. 2004, 59, 5449-5454.

(28) Quadros, P. A.; Baptista, C. M. S. G. Effective interfacial area in agitated liquid-liquid continuous reactors. Chem. Eng. Sci. 2003, 58, 3935-3945.

(29) Quadros, P. A. Nitração de compostos aromáticos: transferência de massa e reacção química, Chapter 8. Ph.D. Thesis, University of Coimbra, Portugal, 2005.

(30) Dummann, G.; Quittmann, U.; Gröschel, L.; Agar, D. W.; Wörz, O.; Morgenschweis, K. The capillary-microreactor: a new reactor concept for the intensification of heat and mass transfer in liquid-liquid reactions. Catal. Today 2003, 79-80, 433-439.

Received for review February 20, 2005 Revised manuscript received May 6, 2005 Accepted May 12, 2005

IE050205M 